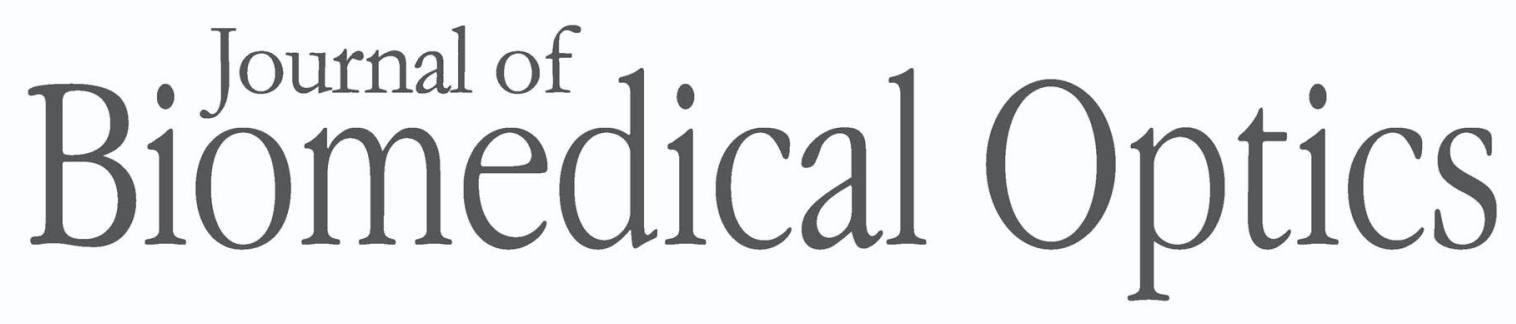

SPIEDigitalLibrary.org/jbo

\title{
In vivo two-photon microscopy to 1.6-mm depth in mouse cortex
}

Demirhan Kobat

Nicholas G. Horton

Chris $\mathrm{Xu}$ 


\title{
In vivo two-photon microscopy to $1.6-\mathrm{mm}$ depth in mouse cortex
}

\author{
Demirhan Kobat, Nicholas G. Horton, and Chris Xu \\ Cornell University, School of Applied and Engineering Physics, Ithaca, New York 14853
}

\begin{abstract}
Deep tissue in vivo two-photon fluorescence imaging of cortical vasculature in a mouse brain using 1280-nm excitation is presented. A record imaging depth of $1.6 \mathrm{~mm}$ in mouse cortex is achieved in vivo, approximately reaching the fundamental depth limit in scattering tissue. ( 2011 Society of Photo-Optical Instrumentation Engineers (SPIE). [DOI: 10.1117/1.3646209]
\end{abstract}

Keywords: microscopy; imaging; multiphoton processes; scattering; fluorescence.

Paper 11337LR received Jun. 30, 2011; revised manuscript received Aug. 28, 2011; accepted for publication Sep. 14, 2011; published online Oct. 21, 2011.

Optical imaging plays a major role in both basic biological research and clinical diagnostics, providing noninvasive or minimally-invasive capability to investigate biological tissue. Optical image acquisition through significant depths of biological tissues, however, presents a major challenge since tissue is heterogeneous and the strong scattering of the various tissue components have historically restricted high-resolution optical imaging to thin sections or to superficial layers. The development of two-photon fluorescence microscopy (TPM) has significantly extended the penetration depth of high-resolution optical imaging. Indeed, the most promising and successful area in biological imaging that showcases the unique advantage of TPM is imaging deep into scattering tissues, ${ }^{1}$ particularly for in vivo applications. In the last 15 years TPM has enabled, in many fields for the first time, direct visualization of the behavior of cells in their natural environment. ${ }^{2}$ For example, TPM has proven to be well-suited for a variety of imaging applications deep within intact or semi-intact tissues, as demonstrated in the studies of neuronal activity ${ }^{3}$ and anatomy, ${ }^{4}$ developing embryos, ${ }^{5}$ and tissue morphology and pathology. 6,7 When compared to one-photon confocal microscopy, a factor of 2 to 3 improvement in penetration depth is obtained.

Despite all previous efforts, however, TPM has so far been restricted to $\sim 1 \mathrm{~mm}$ in penetration depth in scattering tissue. ${ }^{8,9}$ The maximum imaging depth in TPM depends on the ability of excitation light to reach the focus unscatterred (ballistic excitation photons) and emitted fluorescence to reach the detector. It scales linearly with the attenuation length of the excitation light in tissue and logarithmically with the inverse of the duty cycle and the average power incident on the sample surface. ${ }^{1}$ The penetration depth can be improved to a certain extent by reducing the pulse duty cycle, for example, Theer et al. achieved $1 \mathrm{~mm}$ imaging depth with $800-\mathrm{nm}$ excitation by using a Ti:Sapphire regenerative amplifier ( $\mu \mathrm{J}$ pulses) with a repetition rate of $200 \mathrm{kHz} .{ }^{9}$ However, the fundamental depth limit for high resolution TPM is due to the loss of the signal-to-background ratio (SBR) of the excitation in scattering biological tissue. ${ }^{1}$ Numerical and experimental investigations in the past have shown that

Address all correspondence to: Demirhan Kobat, Cornell University, School of Applied and Engineering Physics, Ithaca, New York 14853; Tel: + 1 (607) 255 1624; Fax: + 1 (607) 255 1624; E-mail: dk287@cornell.edu. this fundamental depth limit is reached at approximately 5 to 6 attenuation lengths of the excitation light, ${ }^{10-12}$ which corresponds to approximately 0.8 to $1-\mathrm{mm}$ penetration depth in mouse cortex in vivo at $800 \mathrm{~nm}$. A more effective strategy for improving the imaging depth is reducing the attenuation of excitation light by tissue. As a result of the large difference between scattering mean free paths and absorption lengths in brain tissue, scattering is the dominant attenuation factor over water and intrinsic molecule absorption for wavelengths between 350 and $1300 \mathrm{~nm}$. TPM with longer excitation wavelength enables deeper penetration due to the significantly reduced tissue scattering. ${ }^{8}$ Furthermore, the use of longer excitation wavelengths will typically result in longer wavelength fluorescence emissions, where there is less absorption due to intrinsic molecules, yielding an additional increase in signal collection. There have been previous studies reporting the superior penetration depth of longer excitation wavelengths in tissue models, ${ }^{13}$ as well as in several $e x$ vivo and in vivo tissues. ${ }^{8,14,15}$

In this paper we demonstrate in vivo imaging up to approximately 1.6-mm penetration depth in the cortex of a mouse brain with 1280-nm excitation, using only nanojoule (nJ) pulse energies at the sample surface. Our results significantly extend the previous depth record, and approximately reach the fundamental depth limit for multiphoton imaging in scattering tissue at $1280 \mathrm{~nm}$.

An optical parametric oscillator (OPO, $\lambda_{\text {center }}=1280 \mathrm{~nm}$, $80 \mathrm{MHz}$ repetition rate, $140 \mathrm{fs}$ pulse width, OPAL BB, Spectra Physics), pumped by a mode-locked femtosecond Ti:Sapphire laser (Tsunami, Spectra Physics) is used as the excitation source. The raster-scanned beam is focused into the sample by a high numerical aperture (NA) water immersion lens (Olympus XLMPlan Fluar $20 \times 0.95 \mathrm{NA}$ ). The fluorescence from the sample is epicollected and directed to a photomultiplier tube (PMT) by a dichroic mirror. We use a PMT (H7422-50, Hamamatsu Photonics) with a GaAs photocathode for efficient signal detection beyond $700 \mathrm{~nm}$. A movable objective microscope head (Sutter Instrument Company) translates the objective axially to change the imaging depth within the sample. A computer running MPSCOPE software ${ }^{16}$ controls both the translation of the objective

$1083-3668 / 2011 / 16(10) / 106014 / 4 / \$ 25.00$ C 2011 SPIE 
(a)

\section{(b)}
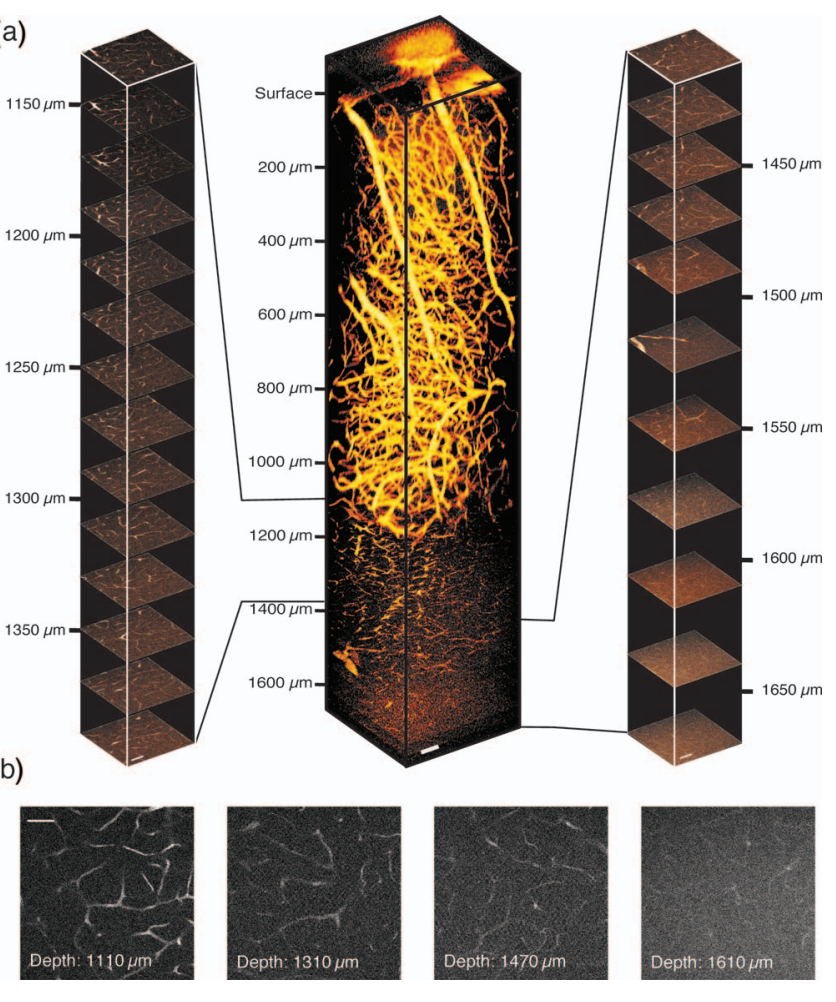

Fig. 1 Two photon fluorescence images of cortical vasculature in a mouse brain. (a) $235 x-y$ frames from $60 \mu \mathrm{m}$ above the cortical surface to $1110 \mu \mathrm{m}$ below are taken at a depth increment of $5 \mu \mathrm{m}$. The depth increments in the stack are $20 \mu \mathrm{m}$ from 1110 to $1490 \mu \mathrm{m}$ and $30 \mu \mathrm{m}$ from 1490 to $1670 \mu \mathrm{m}$. These increments are adjusted to keep the total imaging time at a practical limit while covering all the available depths. 3D reconstruction is made in IMAGE J software using the volume viewer plug-in. Expanded 3D stacks are shown for the deepest sections $(>1130 \mu \mathrm{m})$. (b) Four normalized $x-y$ frames from the $z$-stack at various depths. 8, 16, 32, and 224 frames (at 1 frame/s acquisition speed) were averaged for the sections at imaging depths of 1110,1310,1470, and $1610 \mu \mathrm{m}$, respectively. Scale bars are $50 \mu \mathrm{m}$ for both (a) and (b).

and image acquisition. The power at the sample surface is adjusted by a half-wave plate and a polarization beam splitter cube. The half-wave plate is rotated by a servomotor, which is also controlled by MPSCOPE as explained in Ref. 16. Current pulses generated by the PMT are converted to voltage pulses $(0.1 \mathrm{~V} / \mu \mathrm{A})$ and low-pass filtered $(200 \mathrm{KHz})$ by a commercial preamp (C7319, Hamamatsu Photonics). Analog-to-digital

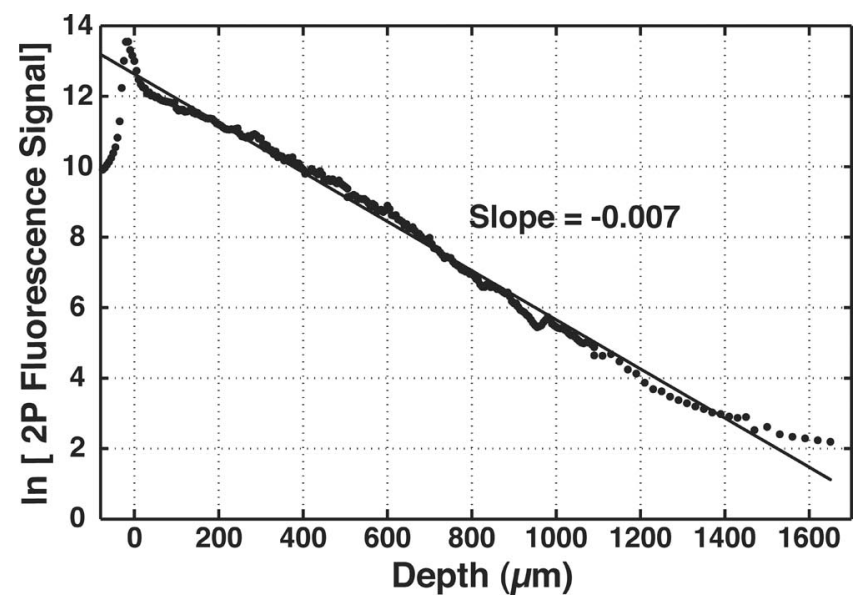

Fig. 2 Fluorescence intensity as a function of imaging depth for the stack shown in Fig. 1. Fluorescence signal strength at a particular depth is represented by the average value of the brightest $1 \%$ of the pixels in the $x-y$ image at that depth.

conversion is performed by a data acquisition card (NI PCI6110 , National Instruments) at a sampling rate up to 5 million samples/s.

We used adult (10 to 12 weeks old) male FVB/N mice for all the experiments in this study. Animals were anesthetized using isoflurane (MWI) (4\% in $\mathrm{O}_{2}$ for induction, $1.5 \%$ to $2 \%$ in $\mathrm{O}_{2}$ for surgery and imaging) and were hydrated with $50 \mu \mathrm{L} / \mathrm{h}$ subcutaneous injections of 5\% glucose (Sigma) in saline (MWI). Body temperature was maintained with a feedback-controlled heat blanket (Harvard Apparatus). Prior to surgery, glycopryllate and dexamethasome were administered by intramuscular injections to the quadriceps. After the skull was exposed and cleaned with ethanol $(70 \%)$ and ferric chloride (10\%, VWR), a custom-built metal plate was attached to the skull using a cyanocrylate-based glue (Loctite-495) and dental cement (A-M Systems). A 4-mm diameter craniotomy was centered either at the Bregma point or at $2 \mathrm{~mm}$ posterior and lateral to the Bregma point. The dura was left intact. A 5-mm diameter glass coverslip (Electron Microscopy Sciences) was used to seal the craniotomy. All animal procedures were reviewed and approved by the Cornell Institutional Animal Care and Use Committee.

$100 \mu \mathrm{l}$ of $10 \mathrm{kDa}$ Alexa680-Dextran (Invitrogen, dissolved at $5 \% \mathrm{w} / \mathrm{v}$ in saline) was retro-orbitally injected to label the vasculature. Imaging sites were approximately $0.5 \mathrm{~mm}$ anterior and (a)

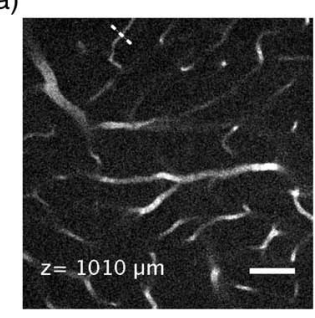

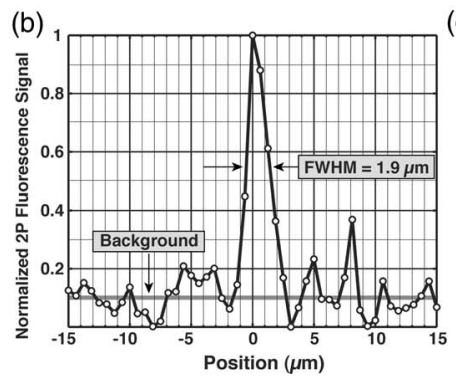

Fig. 3 Intensity line profiles across lateral blood vessels are used to characterize the SBR and resolution. (a) A sample $x-y$ frame at $1010 \mu \mathrm{m}$ depth, where a line profile (b) is taken along the dashed line. The scale bar is $50 \mu \mathrm{m}$. (b) The FWHM of the profiles presents an upper bound for the lateral resolution. Background is calculated by averaging the values between -15 and $-5 \mu \mathrm{m}$ and between 5 and $15 \mu \mathrm{m}$. (c) The SBR as a function of depth. 


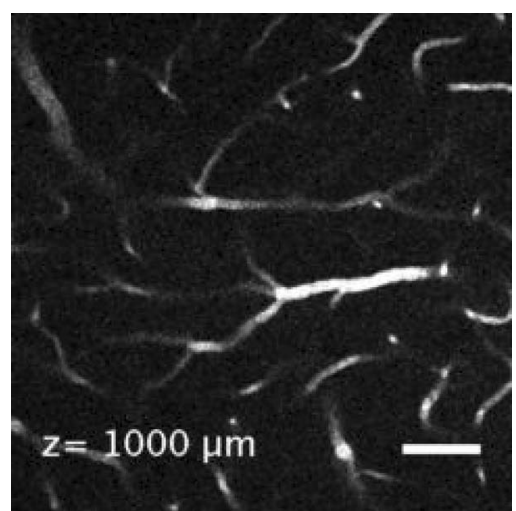

Video 1 Full z-stack of two photon fluorescence images of mouse cortical vasculature shown in Fig. 1. All frames are normalized. The scale bar is $50 \mu \mathrm{m}$. (QuickTime, 4.6 MB)

[URL: http://dx.doi.org/10.1117/1.3646209.1]

lateral to bregma or $2 \mathrm{~mm}$ posterior and lateral to bregma for the deep cortical stack $(\sim 1.6 \mathrm{~mm})$ and the shallower stack $(\sim 1 \mathrm{~mm})$, respectively. We acquired images with a size of $512 \times 512$ pixels, with a pixel dwell time of $3.05 \mu \mathrm{s}$. MPSCAN acquired data during the middle $80 \%$ of the scan path on the fast axis, leading to an image acquisition rate of 1 frame per second (fps). For the top 0.7-mm imaging depth, the excitation powers were adjusted so that approximately the same signal level and thus the same signal-to-noise ratio (SNR) could be achieved at a constant frame rate of $1 \mathrm{fps}$, starting with $20 \mathrm{~mW}$ for the surface level imaging. At the imaging depths beyond $0.7 \mathrm{~mm}$, the full output power of the OPO was used, which corresponded to approximately $120-\mathrm{mW}$ (i.e., $1.5-\mathrm{nJ}$ pulse energy) average power at the surface of the brain. Imaging integration times were then increased in accordance with the imaging depths to maintain reasonably constant SNRs throughout the imaging depth of $1.6 \mathrm{~mm}$.

Figure 1(a) shows the three-dimensional (3D) reconstruction of the cortical vasculature of a mouse. An imaging depth of approximately $1.6 \mathrm{~mm}$ is achieved, significantly extending the previous depth record of $1 \mathrm{~mm}$ for in vivo multiphoton imaging in a mouse brain. $Z$-sections ( $x-y$ frames) at various depths are shown in Fig. 1(b) (for the entire $z$-stack see Video 1). Microscopic features of the mouse brain vasculature are still discernable at $1.6 \mathrm{~mm}$ below the surface of the brain. While a long integration time is currently necessary for achieving an acceptable SNR at the greatest depths, the integration time can be significantly reduced by using more energetic laser pulses to enhance the nonlinearly generated signal without increasing the average laser power at the surface of the brain. This will also enable fast monitoring of tissue dynamics, such as measuring vascular circulation speeds.

Figure 2 shows the detected fluorescence signal as a function of imaging depth. A fluorescence signal attenuation length (i.e., the depth at which the fluorescence signal attenuates by $1 / \mathrm{e}^{2}$ ) of approximately $0.3 \mathrm{~mm}$ is measured, which is consistent with the values reported in previous studies. ${ }^{8} \mathrm{Up}$ to $1.4-\mathrm{mm}$ imaging depth, the attenuation length remains remarkably constant over more than 4 orders of magnitude in signal strength. At beyond $1.4 \mathrm{~mm}$, the attenuation curve flattens somewhat, which is (a)

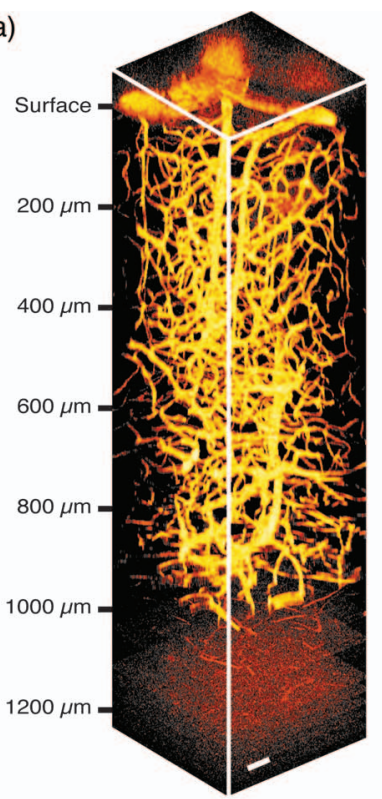

(b)
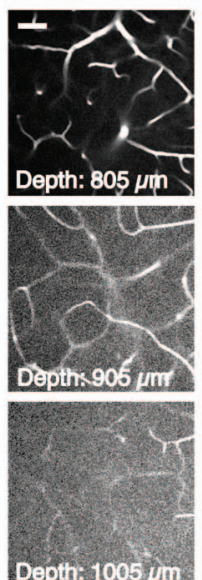

(c)

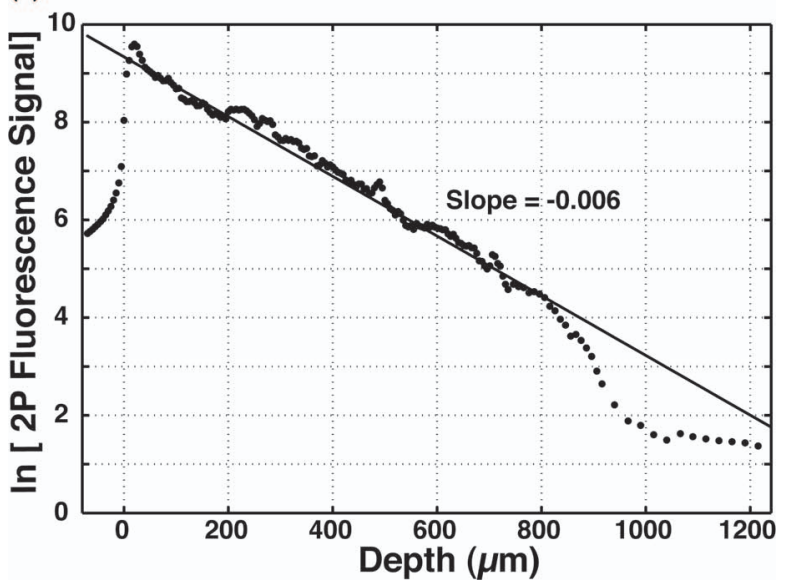

Fig. 4 Two photon fluorescence images of mouse cortical vasculature at approximately $2 \mathrm{~mm}$ posterior and lateral to bregma, where the cortical thickness is approximately $800 \mu \mathrm{m}$. (a) $161 x-y$ frames from $80 \mu \mathrm{m}$ above the cortical surface to $725 \mu \mathrm{m}$ below were taken at a depth increment of $5 \mu \mathrm{m}$. For the top $635-\mu \mathrm{m}$ imaging depth, the excitation powers were adjusted so that approximately the same SNR can be achieved at a constant frame rate of 1 frame/s. At the imaging depths beyond $635 \mu \mathrm{m}$, the full output power of the OPO $(120 \mathrm{~mW}$ at the brain surface) was used. Imaging integration times were then increased in accordance with the imaging depths to maintain reasonably constant SNRs throughout the imaging depth of $1.2 \mathrm{~mm}$. The depth increments in the stack were $10 \mu \mathrm{m}$ from 725 to $905 \mu \mathrm{m}$ and $25 \mu \mathrm{m}$ from 905 to $1230 \mu \mathrm{m}$. 3D reconstruction was made in IMAGE J software using the volume viewer plug-in. (b) Four normalized $x-y$ frames from the $z$-stack at various depths. 16 frames (at 1 frame/s acquisition speed) were averaged for the sections at imaging depths of 805 and $905 \mu \mathrm{m}$. Thirty frames were averaged for the sections at imaging depths of 1005 and $1105 \mu \mathrm{m}$. Scale bars are $50 \mu \mathrm{m}$ for both (a) and (b). (c) Fluorescence intensity as a function of imaging depth for the stack shown in (a). Fluorescence signal strength at a particular depth is represented by the average value of the brightest $1 \%$ of the pixels in the $x-y$ image at that depth. For the first 800- $\mu \mathrm{m}$ imaging depth (approximately the cortical thickness), a constant fluorescence signal attenuation length of approximately $330 \mu \mathrm{m}$ is observed. However, beyond $800 \mu \mathrm{m}$ an abrupt change of the slope is present since the tissue beneath the gray matter is significantly more scattering. A rapid decrease of SBR and SNR is observed in $x-y$ images at this region, as shown in (b). 
caused by the diminishing SBR at these depths, and is an indication of reaching the SBR limit. In order to quantify the decrease in SBR we used the intensity line profiles across lateral blood vessels, such as the one shown in Figs. 3(a) and 3(b). While the peak value of the profile is the sum of the signal and the background, the average intensity in the region immediately outside of the vessel gives a measure of the background fluorescence generated outside the focal volume. We used the $10-\mu \mathrm{m}$ long portion of the line on each side, starting $5 \mu \mathrm{m}$ away from the center of the vessel, to calculate the background value. Figure 3(c) shows the SBR calculated using this method at different depths. The SBR decreases with depth and reaches the practical limit of 1 at approximately $1.5-\mathrm{mm}$ depth. This finding is corroborated by the fact that more than 5 attenuation lengths is reached in our experiments, a limit that was also observed previously by other research groups using shorter excitation wavelengths and at much shallower imaging depth. ${ }^{9}$ Since the thickness of the cortex in many areas of the mouse brain is less than $1.6 \mathrm{~mm},{ }^{17}$ our results clearly show that $1280-\mathrm{nm}$ excitation is capable of imaging through the entire cortex layer of the mouse in vivo (an example is shown in Fig. 4).

The theoretical value of the full width half maximum (FWHM) of the two-photon point spread function (PSF) is $0.51 \mu \mathrm{m}$ in the lateral dimension for a 0.95 -NA water immersion lens and 1280-nm excitation. Even the smallest blood vessels (2 to $3 \mu \mathrm{m}$ in diameter) are not subresolution; therefore we cannot use them to determine the exact PSF. However, the line profiles across these thin blood vessels also provide an upper bound estimate for lateral resolution. We were able to obtain less than $3 \mu \mathrm{m}$ FHWM in the line profiles up to $1.5 \mathrm{~mm}$ imaging depth. For example, the intensity profile across the blood vessel shown in Fig. 3 had a FWHM of $1.9 \mu \mathrm{m}$. Thus, no loss of resolution in the lateral dimension is observed in our experiment. However, in an image stack (not shown) obtained with 1280-nm excitation through a 0.6-NA water immersion lens (theoretical two-photon $\mathrm{PSF}$ axial $\mathrm{FWHM}=5.6 \mu \mathrm{m}$ ) and $0.4-\mu \mathrm{m} z$-steps, we measured the FWHM of axial intensity profiles as 7, 9, and $12 \mu \mathrm{m}$ at depths of 230, 435, and $900 \mu \mathrm{m}$, respectively. This result implies that inclusion of an adaptive optics device for aberration correction will potentially improve the resolution and SBR at 1280-nm excitation. ${ }^{18}$

In summary, we demonstrate in vivo deep tissue TPM of a mouse brain using 1280-nm excitation. A record imaging depth of $1.6 \mathrm{~mm}$ in a mouse cortex is achieved, approximately reaching the fundamental depth limit for TPM. Our results will greatly facilitate in vivo studies of intact brain at depths that are significantly beyond what was previously reachable.

\section{Acknowledgments}

This research project is supported by NSF/DBI (Grant No. DBI0546227; C.X.). Nicholas G. Horton is supported by NSF Graduate Research Fellowship (Grant No. DGE-0707428). We would like to thank Chris B. Schaffer, Nozomi Nishimura, and Daniel Dombeck for discussions and technical suggestions.

\section{References}

1. F. Helmchen and W. Denk, "Deep tissue two-photon microscopy," Nat. Methods 2(12), 932-940 (2005).

2. K. Svoboda, F. Helmchen, W. Denk, and D. W. Tank, "Spread of dendritic excitation in layer $2 / 3$ pyramidal neurons in rat barrel cortex in vivo," Nat. Neuroscience 2(1), 65-73 (1999).

3. D. Dombeck, A. Khabbaz, F. Collman, T. Adelman, and D. Tank, "Imaging large-scale neural activity with cellular resolution in awake, mobile mice," Neuron 56, 43-57 (2007).

4. R. Mostany and C. Portera-Cailliau, "Absence of large-scale dendritic plasticity of layer 5 pyramidal neurons in peri-infarct cortex," J. Neurosci. 31(5), 1734-1738 (2011).

5. T.-H. Tsai, C.-Y. Lin, H.-J. Tsai, S.-Y. Chen, S.-P. Tai, K.-H. Lin, and C.-K. Sun, "Biomolecular imaging based on far-red fluorescent protein with a high two-photon excitation action cross section," Opt. Lett. 31(7), 930-932 (2006).

6. R. M. Williams, A. Flesken-Nikitin, L. H. Ellenson, D. C. Connolly, T. C. Hamilton, A. Y. Nikitin, and W. R. Zipfel, "Startegies for highresolution imaging of epithelial ovarian cancer by laparoscopic nonlinear microscopy," Transl. Oncol. 3(3), 181-194 (2010).

7. R. Yadav, S. Mukherjee, M. Hermen, G. Tan, F. R. Maxfield, W. W. Webb, and A. K. Tewari, "Multiphoton microscopy of prostate and periprostatic neural tissue: a promising imaging technique for improving nerve-sparing prostatectomy," J. Endourol. 23(5), 861-867 (2009).

8. D. Kobat, M. E. Durst, N. Nishimura, A. W. Wong, C. B. Schaffer, and C. $\mathrm{Xu}$, "Deep tissue multiphoton microscopy using longer wavelength excitation," Opt. Express 17(16), 13354-13364 (2009).

9. P. Theer, M. Hasan, and W. Denk, "Two-photon imaging to depth of $1000 \mu \mathrm{m}$ in living brains by use of a Ti: $\mathrm{Al}_{2} \mathrm{O}_{3}$ regenerative amplifier," Opt. Lett. 28(12), 1022-1024 (2003).

10. A. Leray, C. Odin, E. Huguet, F. Amblard, and Y. Le Grand, "Spatially distributed two-photon excitation fluorescence in scattering media: Experiments and time-resolved Monte Carlo simulations," Opt. Commun. 272, 269-278 (2007).

11. M. Oheim, E. Beaurepaire, E. Chaigneau, J. Mertz, and S. Charpak, "Two-photon microscopy in brain tissue: Parameters influencing the imaging depth," J. Neurosci. Methods 111, 29-37 (2001).

12. P. Theer and W. Denk, "On the fundamental imaging-depth limit in twophoton microscopy," J. Opt. Soc. Am. A 23(12), 3139-3149 (2006).

13. M. Balu, T. Baldacchini, J. Carter, T. B. Krasieva, R. Zadoyan, and B. J. Tromberg, "Effect of excitation wavelength on penetration depth in nonlinear optical microscopy of turbid media," J. Biomed. Opt. 14(1), 010508 (2009).

14. V. Andresen, S. Alexander, W.-M. Heupel, M. Hirschberg, R. M. Hoffman, and P. Friedl, "Infrared multiphoton microscopy: Subcellularresolved deep tissue imaging," Curr. Opin. Biotechnol. 20, 54-62 (2009).

15. J. Herz, V. Siffrin, A. E. Hauser, A. U. Brandt, T. Leuenberger, H. Radbruch, F. Zipp, and R. A. Niesner, "Expanding two-photon intravital microscopy to the infrared by means of optical parametric oscillator," Biophys. J. 98, 715-723 (2010)

16. Q. Nguyen, P. S. Tsai, and D. Kleinfeld, "Mpscope: A versatile software suite for multiphoton microscopy," J. Neurosci. Methods 156, 351-359 (2006).

17. K. B. J. Franklin and G. Paxinos, The Mouse Brain in Stereotaxic Coordinates, 3rd Ed., Academic Press, New York (2007).

18. J. M. Girkin, S. Poland, and A. J. Wright, "Adaptive optics for deeper imaging of biological samples," Curr. Opin. Biotechnol. 20, 106-110 (2009). 